\title{
Alternative Mechanism of Aspirin in Anti-Thrombotic Therapy: Inhibition of Thrombin Activatable Fibrinolysis Inhibitor
}

\author{
Seong Soo A. An ${ }^{*}$ and Robert S. Greenfield ${ }^{\dagger}$ \\ Department of Bionano Technology, Gachon Bionano Research Institute, Gachon University, Sungnam, \\ Gyeonggi-do 461-701, Korea. E-mail: seong.an@gmail.com \\ ${ }^{\dagger}$ Department of Research and Development, American Diagnostica Inc., Stamford, CT, USA \\ Received February 20, 2012, Accepted June 26, 2012
}

\begin{abstract}
The use of aspirin is widely recommended for the prevention of heart attacks owing to its ability to inhibit platelet activation by irreversibly blocking cyclooxygenase 1 . However, aspirin also affects the fibrinolytic and hemostatic pathways by mechanisms that are not well understood, causing severe hemorrhagic complications. Here, we investigated the ability of aspirin and aspirin metabolites to inhibit thrombin-activatable fibrinolysis inhibitor (TAFI), the major inhibitor of plasma fibrinolysis. TAFI is activated via proteolytic cleavage by the thrombin-thrombomodulin complex to TAFIa, a carboxypeptidase B-like enzyme. TAFIa modulates fibrinolysis by removing the C-terminal arginine and lysine residues from partially degraded fibrin, which in turn inhibits the binding of plasminogen to fibrin clots. Aspirin and its major metabolites, salicylic acid, gentisic acid, and salicyluric acid, inhibit TAFIa carboxypeptidase activity. Salicyluric acid effectively blocks activation of TAFI by thrombin-thrombomodulin; however, salicylates do not inhibit carboxypeptidase $\mathrm{N}$ or pancreatic carboxypeptidase B. Aspirin and other salicylates accelerated the dissolution of fibrin clots and reduced thrombus formation in an in vitro model of fibrinolysis. Inhibition of TAFI represents a novel hemostatic mechanism that contributes to aspirin's therapy-associated antithrombotic activity and hemorrhagic complications.
\end{abstract}

Key Words : Aspirin, Thrombin activatable fibrinolysis inhibitor (TAFI), Fibrinolysis

\section{Introduction}

Aspirin (acetylsalicylic acid, ASA), one of the oldest known drugs, is one of the most widely used over-thecounter medications for the treatment of pain and inflammation. The antithrombotic effect of aspirin has long been recognized, and low doses of aspirin are recommended for the prevention of ischemic events in patients with coronary artery disease (CAD). The use of aspirin alone or in combination with other antithrombotics significantly reduces the incidence of cardiovascular death, stroke, and myocardial infarction. In large clinical studies, aspirin significantly reduced the occurrence of myocardial infarction and the mortality rate of patients with unstable angina pectoris. ${ }^{1-3}$ Recent studies have shown that aspirin given shortly after coronary artery bypass surgery also greatly reduced the mortality, stroke, and other ischemic complications. ${ }^{4}$ Aspirin therapy is now generally considered routine practice and has become the most widely prescribed prophylactic antiplatelet drug owing to its efficacy and low cost.

The action of aspirin and other nonsteroidal anti-inflammatory drugs (NSAIDs), such as indomethacin, acetaminophen, and ibuprofen, is thought to be mostly derived from the selective inhibition of cyclooxygenases 1 and 2 (COX-1 and COX-2). COX-1 catalyzes the synthesis of thromboxane A2 (Tx-A2), which causes platelet activation, vasoconstriction, and smooth muscle proliferation. ${ }^{5,6}$ Aspirin at low doses selectively and irreversibly inhibits COX-1 and blocks the formation of Tx-A2. Vane et al. ${ }^{7}$ showed that by blocking the synthesis of prostaglandins, aspirin prevented blood platelets from aggregating, one of the initial steps in the formation of blood clots. At higher doses, aspirin inhibits COX-2, which controls the synthesis of prostacyclin, another regulator of platelet function, and mediates antiinflammatory, analgesic, and anticancer effects. ${ }^{8}$

Aspirin has been shown to synergize antithrombotic therapy with a tissue plasminogen activator (tPA) or a streptokinase treatment for acute myocardial infarction, ${ }^{9}$ although gastric and cerebral hemorrhages are significant and dangerous complications. ${ }^{10}$ However, antiplatelet activity cannot fully account for all the beneficial antithrombotic activities of aspirin. ${ }^{11}$ Evidence indicates that the beneficial effects of aspirin may also be mediated by direct effects on the coagulation and fibrinolytic pathways. Aspirin was found to reduce thrombin generation at the site of injury and affect tissue factor-initiated coagulation. ${ }^{12}$ Enhanced plasma fibrinolysis has frequently been demonstrated during aspirin therapy. ${ }^{13-16}$

The mechanism by which aspirin alters fibrinolysis has not been fully elucidated. Low-dose aspirin was found to leave the fibrinolytic capacity of the vessel wall intact ${ }^{17}$ and increase fibrin gel porosity in vitro. ${ }^{18}$ Enhanced fibrinolysis associated with aspirin administration was independent of tPA levels and may be associated with fibrinogen acetylation. ${ }^{19}$ Aspirin-induced alterations in the fibrinolytic pathway may act synergistically with antiplatelet effects to 


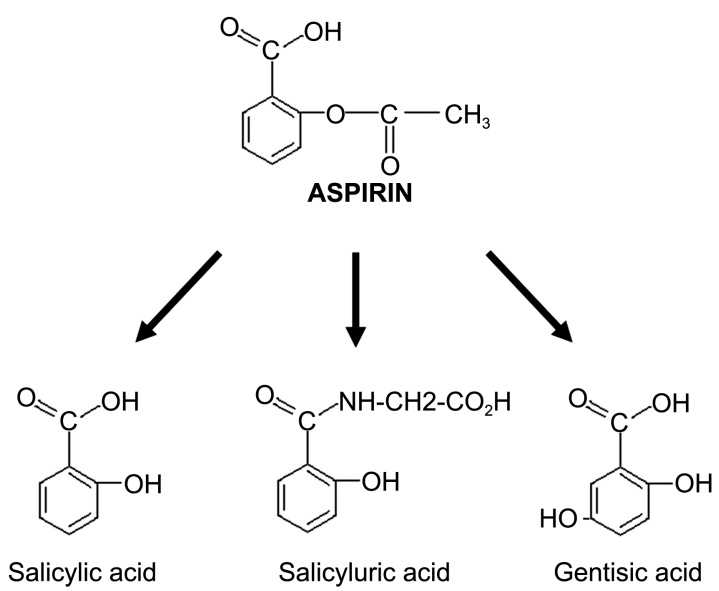

Figure 1. Structure of aspirin and its major metabolites.

promote antithrombotic activity. Unfortunately, induction of profibrinolytic activity could also lead to an imbalance in hemostasis and increase the likelihood of dangerous gastric and cerebral bleeding side effects.

Thrombin-activatable fibrinolysis inhibitor (TAFI), also called plasma procarboxypeptidase $\mathrm{B}$ or $\mathrm{U}$, is a $60-\mathrm{kD}$ plasma protein that plays an important role in regulation of the balance between fibrinolysis and coagulation. ${ }^{20,21}$ TAFI is converted to an active carboxypeptidase enzyme (TAFIa, $35 \mathrm{kD})$ via proteolytic cleavage by the thrombin-thrombomodulin (TH/TM) complex or plasmin..$^{22,23}$ TAFIa modulates fibrinolysis by removing $\mathrm{C}$-terminal lysine residues from partially degraded fibrin, which inhibits the binding of plasminogen to fibrin clots and results in a reduced rate of fibrinolysis. Changes in plasma TAFI levels have been reported in various pathological conditions including liver disease, ${ }^{24}$ leukemia, ${ }^{25}$ disseminated intravascular coagulation, ${ }^{26} \mathrm{CAD},{ }^{27}$ and deep vein thrombosis. ${ }^{28}$ Alterations of fibrinolysis in hemophilia have recently been associated with enhanced plasmin-induced TAFI activation. ${ }^{29-32}$ Inhibition of the carboxypeptidase activity of TAFI has been shown to modulate fibrinolysis in vivo and alter hemostatic balance toward a profibrinolytic state. ${ }^{33}$

In this report, we investigated whether aspirin-induced alterations in fibrinolytic activity are mediated by TAFI inhibition. We studied the effects of aspirin and major aspirin metabolites (Figure 1) on TAFIa carboxypeptidase activity and on TH/TM-induced TAFI activation. Aspirin and various other salicylates (but not acetaminophen or ibuprofen) inhibited TAFIa carboxypeptidase activity. Salicyluric acid (SU) was found to inhibit TH/TM-induced TAFI activation. Salicylates that inhibited TAFI were also tested in in vitro models of thrombosis and fibrinolysis. We propose the use of aspirin and its metabolic derivatives to inhibit TAFI as a novel mechanism that leads to enhanced fibrinolytic activity. Drug-induced increased fibrinolysis can augment the beneficial antithrombotic activity of aspirin and potentiate the dangerous bleeding complications associated with aspirin therapy.

\section{Experimental}

Materials. Human TAFI was purchased from Hematologic Technologies Inc. (Essex Junction, VT). TAFIa, thrombin and thrombomodulin were from American Diagnostica Inc. (Stamford, CT). TAFIa carboxypeptidase activity was determined using ACTIFLUOR ${ }^{\mathrm{TM}}$ TAFI, a fluorescencebased assay from American Diagnostica Inc. (Stamford, CT). ASA, 3-salicylic acid (SA3), salicylic acid (SA), gentisic acid (GA), SU, ibuprofen, acetaminophen and porcine pancreatic carboxypeptidase B were obtained from Sigma Chemical Co. (St. Louis, MO).

Inhibition of TAFIa. TAFIa (50 ng) was preincubated with and without the indicated drugs in $200 \mathrm{mM}$ Tris buffer, $\mathrm{pH} 7.4$ for various periods of time at room temperature. Residual carboxypeptidase activity was determined using the components from ACTIFLUOR TAFI kit. Substrate A $(30 \mu \mathrm{L})$ and Substrate B $(30 \mu \mathrm{L})$ were added to TAFIa aliquots $(160 \mu \mathrm{L})$ in microtiter wells. The reaction was ran for $40 \mathrm{~min}$ and stopped with $50 \mu \mathrm{L}$ of the Fluorescence Quencher. Fluorescence was measured in a SpectroMax Spectrofluorometer (Molecular Devices, CA) at $\varepsilon_{\mathrm{ex}}=563$ $\mathrm{nm}$ and $\varepsilon_{\mathrm{em}}=590 \mathrm{~nm}$.

Effects of Salicylates on Activation of TAFI by Thrombin/Thrombomodulin. Test compounds were dissolved in $20 \mathrm{mM}$ Tris-HCl, $\mathrm{pH} 7.4$ and added into the microtiter wells containing TAFI. Thrombin/thrombomodulin was added to the wells, and the activity of TAFIa was monitored by removing $20 \mu \mathrm{L}$ aliquots from microtiter wells and measuring residual TAFIa activity.

Analysis of TAFI Activation by SDS-PAGE. TAFI and thrombin/thrombomodulin complex was incubated with various salicylate compounds for 30 minutes at $37^{\circ} \mathrm{C}$. The reaction mixtures were applied onto sodium dodecyl sulfatepolyacrylamide gel electrophoresis (SDS-PAGE; 5-20\%). The gels were stained using silver stain or Coomassie Blue.

Clot Formation Assay. Test compounds $(50 \mu \mathrm{L})$ at various concentrations were mixed with normal human plasma $(100 \mu \mathrm{L})$ in microtiter wells. Initiation of clot formation was done by adding $10 \mu \mathrm{L}$ of $5 \mathrm{mM} \mathrm{CaCl}_{2}, 15 \mu \mathrm{L}$ of thrombin $(15 \mathrm{ug} / \mathrm{mL})$ and rabbit thrombomodulin $(1.6 \mathrm{U} /$ $\mathrm{mL})$. The change in turbidity was monitored at $340 \mathrm{~nm}$ during incubation at $37^{\circ} \mathrm{C}$ over 20 minutes.

Clot Lysis Assay. Test compounds $(50 \mu \mathrm{L})$ or buffer (50 $\mu \mathrm{L}$ as control) were added to $100 \mu \mathrm{L}$ normal human plasma into microtiter plate wells. The clots were formed by adding $5 \mathrm{mM} \mathrm{CaCl}_{2}(10 \mu \mathrm{L})$ and $15 \mu \mathrm{L}$ of a complex of thrombin $(15 \mathrm{ug} / \mathrm{mL})$ and rabbit thrombomodulin $(1.6 \mathrm{U} /$ $\mathrm{mL}$ ). Clot formation was monitored at $37{ }^{\circ} \mathrm{C}$ for 20 minutes at $405 \mathrm{~nm}$ in a Spectromax 190 microtiter plate reader (Molecular Devices Corp., Menlo Park, CA). Human tPA (100 ng) and was added to the clot and fibrinolysis was monitored by observing the changes in the turbidity of the clot at $340 \mathrm{~nm}$. The time $\left(\mathrm{t}^{1 / 2}\right)$ to achieve $50 \%$ of the dissolution of clot at a given concentration of test compound was determined. 

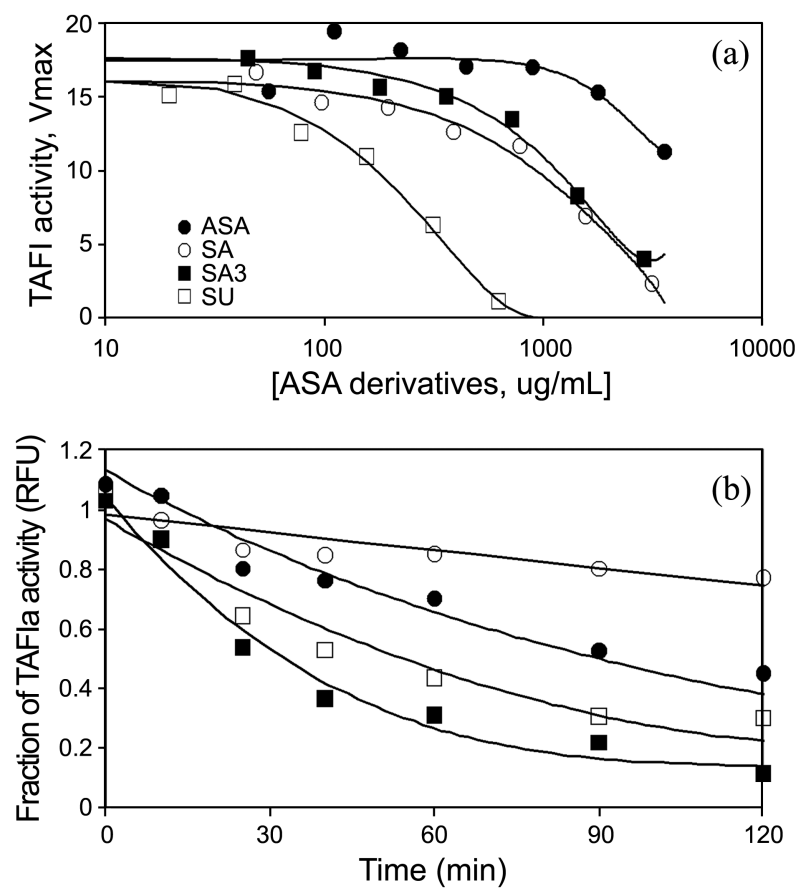

Figure 2. A. Thrombin-activatable fibrinolysis inhibitor a (TAFIa) was preincubated with various concentrations of aspirin (-), salicylic acid (O), 3-salicylic Acid ( $\square$ ), and salicyluric acid ( $\square$ ) for 1 hour at $4{ }^{\circ} \mathrm{C}$, and the residual carboxypeptidase activity was determined using a fluorescence assay. B. Effect of incubation time with salicyluric acid on TAFIa by measuring its activity with its fluorescence substrate. Control (O), $200 \mathrm{mM}(\bullet), 400 \mathrm{mM}$ $(\square)$, and $800 \mathrm{mM}(\boldsymbol{\square})$. The control was referred as TAFIa with buffer and its substrate. The unit was in relative fluorescence unit (RFU), which was normalized to the initial activity.

\section{Results}

Effects of Aspirin and Salicylates on TAFIa Activity. TAFIa activity was significantly reduced in a concentrationdependent manner when the enzyme was preincubated with aspirin and its derivatives (Fig. 2(a)). Aspirin had no apparent effect on TAFIa activity when it was added to the enzymatic substrate without preincubation. The 3 major aspirin metabolites were also evaluated for their ability to inhibit TAFIa. As shown in Table 1, SU was the most potent inhibitor with preincubation with TAFIa, followed by SA3 and SA. The effect of preincubation time on TAFIa activity at various SU concentrations is shown in Figure 2(b). As the SU concentration increased, the TAFIa inactivation rate increased as well. TAFIa carboxypeptidase activity was not

Table 1. Inhibition of TAFIa by aspirin and other salicylates

\begin{tabular}{cc}
\hline Compounds & $\mathrm{K}_{\mathrm{i}}(\mathrm{mM})$ \\
\hline Aspirin $^{a}$ & $6.29 \pm 0.43$ \\
Salicylic Acid $^{a}$ & $7.96 \pm 1.02$ \\
3-Salicylic Acid & \\
Salicyluric Acid $^{a}$ & $6.21 \pm 0.37$ \\
Gentisic Acid $^{b}$ & $1.02 \pm 0.34$ \\
\hline
\end{tabular}

${ }^{a}$ Preincubation for $1 \mathrm{hr}$ with TAFIa prior to assay. ${ }^{b}$ No preincubation

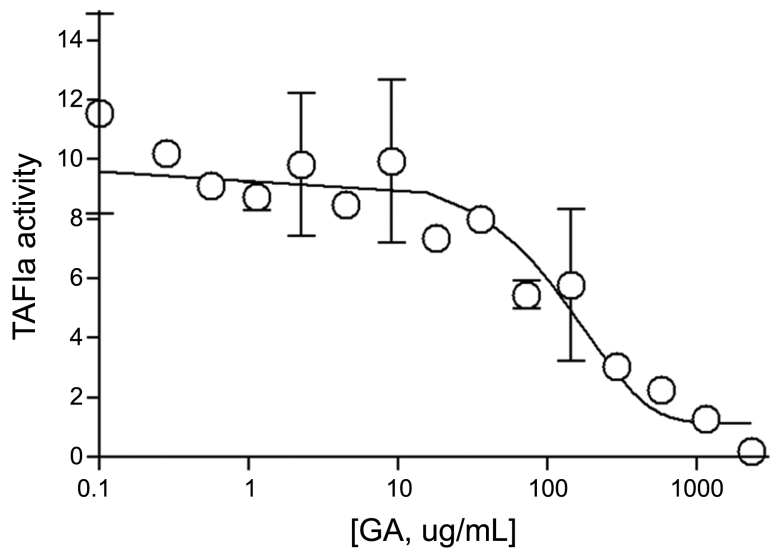

Figure 3. Activity of Thrombin-activatable fibrinolysis inhibitor (TAFI) was measured after the activation by thrombin-thrombomodulin (TH/TM) in the various concentrations of gentisic acid.

significantly reduced without preincubation of the enzyme with SU, SA3, or SA (data not shown). These findings indicate that aspirin and aspirin metabolites are noncompetitive inhibitors of TAFIa. We found little or no effect upon the preincubation of TAFIa with 2 other NSAIDs, ibuprofen and acetaminophen (data not shown), suggesting that salicylates specifically inhibit TAFIa activity.

Gentisic Acid is a Competitive Inhibitor of TAFIa. Gentisic acid, another major metabolite of aspirin, was found to interact differently with TAFIa than the other salicylates. The simultaneous addition of GA and the TAFIspecific fluorescence substrate to TAFIa resulted in a concentration-dependent inhibition of carboxypeptidase activity. GA was an effective competitive inhibitor of TAFIa, with a Ki of $120 \mu \mathrm{M}$ (Table 1, Figure 3). The number and position of the hydroxyl groups on the benzoic acid ring of the salicylates influenced drug binding to TAFIa and inhibition of carboxypeptidase activity.

Effect of Salicylates, Ibuprofen, and Acetaminophen on TH/TM-Induced TAFI Activation. The above-mentioned results suggest that different salicylates may specifically bind to TAFI in different ways or at different site(s). We investigated whether the ability of the TH/TM complex to proteolytically cleave TAFI to TAFIa may be affected by various salicylates or NSAIDs. Aspirin or other salicylates were added to TAFI, and the TH/TM complex and proteolytic cleavage products were analyzed using SDS-PAGE (Fig. 4(a)). TAFI (60 kD) was almost completely converted to TAFIa $(35.8 \mathrm{kD})$ by incubation with the TH/TM complex (lane 7). When SA was added to the activation mixture (lane 2), a major new TAFI proteolytic degradation species (approximately $55 \mathrm{kD}$ ) was generated in addition to TAFIa. This protein was not generated in the TH/TM control. This $55-\mathrm{kD}$ proteolytic product was also observed in lesser amounts when aspirin (lane 1) or gentisic acid (lane 3) was added to the mixture. However, a different proteolytic degradation pattern was seen upon the addition of SU to the TAFI activation mixture. SU inhibited the proteolytic cleavage of TAFI to TAFIa by TH/TM (lane 4), and little if any of 


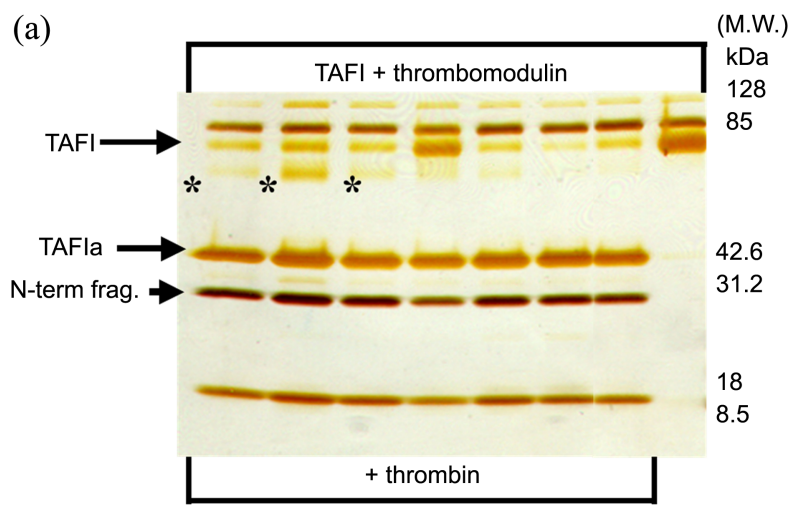

(b)

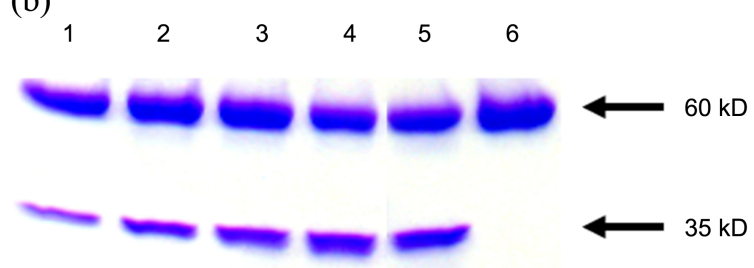

Figure 4. A. Thrombin-activatable fibrinolysis inhibitor (TAFI) was activated by thrombin-thrombomodulin (TH/TM) in the presence of various compounds. After 20 minutes, aliquots were removed, analyzed using sodium dodecyl sulfate-polyacrylamide gel electrophoresis, and performed silver staining. Lane 1, acetylsalicylic acid; 2, salicylic acid; 3, gentisic acid; 4, salicyluric acid; 5 , ibuprofen; 6 , acetaminophen; 7 , TAFI; 8 , TAFI with no TH. *indicated the unusual TAFI activation by thrombin/ thrombomodulin from binding of aspirin onto TAFI. B. Effect of different concentrations of salicyluric acid on TAFI activation by TH/TM from SDS-PAGE, stained with Coomassie blue. Lane 1, $42 \mu \mathrm{g} / \mathrm{mL} ; 2,21 \mu \mathrm{g} / \mathrm{mL} ; 3,10 \mu \mathrm{g} / \mathrm{mL} ; 4,5 \mu \mathrm{g} / \mathrm{mL} ; 5$, control; 6, TAFI with no TH/TM.

the $55-\mathrm{kD}$ species was generated. Ibuprofen (lane 5) and acetaminophen (lane 6) had no significant effect on TAFI cleavage by TH/TM. In a follow-up experiment, inhibition of TH/TM-induced cleavage of TAFI was dependent on the SU concentration (Fig. 4(b)). Thus, the binding of various salicylates can affect the conversion of TAFI to TAFIa by the TH/TM complex and inhibit carboxypeptidase activity.

Effect of Salicylates on an in vitro Fibrinolysis Model. The biochemical studies described previously clearly show that aspirin and various salicylates can inhibit TAFI activation and/or directly inhibit TAFIa carboxypeptidase activity. We investigated whether aspirin or related salicylates can inhibit the antifibrinolytic function of TAFI in an in vitro

Table 2. Enhancement of fibrinolysis by aspirin and other salicylates

\begin{tabular}{cr}
\hline Compounds & \multicolumn{1}{c}{$\mathrm{t}_{\frac{2}{2}(\mathrm{mM})}$} \\
\hline Aspirin & $13.40 \pm 3.11$ \\
Salicylic Acid & $9.44 \pm 4.53$ \\
3-Salicylic Acid & $22.01 \pm 6.14$ \\
Salicyluric Acid & $8.70 \pm 3.81$ \\
Gentisic Acid & $19.80 \pm 4.96$ \\
PTCI & $0.24 \pm 0.13$ \\
\hline
\end{tabular}

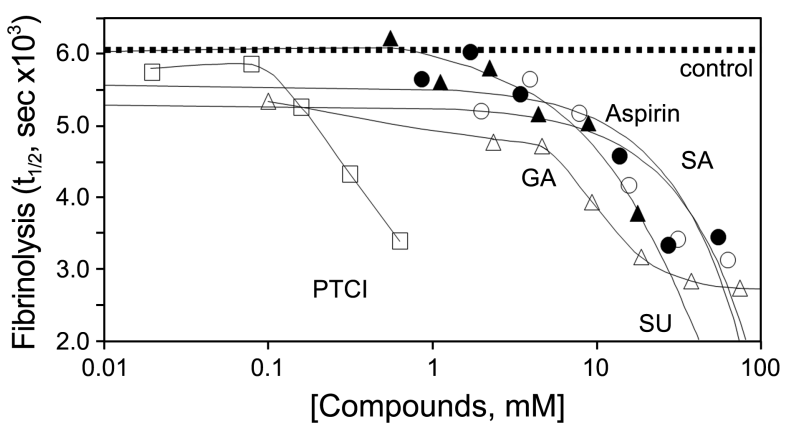

Figure 5. Effects of aspirin, salicylates, and nonsteroidal antiinflammatory drugs on in vitro fibrinolysis. Aspirin (๑), salicyluric acid $(\boldsymbol{\Delta})$, or salicylic acid $(O)$, and gentisic acid $(\Delta)$ were added to the fibrinolysis assay. Potato tuber carboxypeptidase inhibitor $(\square)$ was used as a positive control. The control indicated the negative control with buffer only.
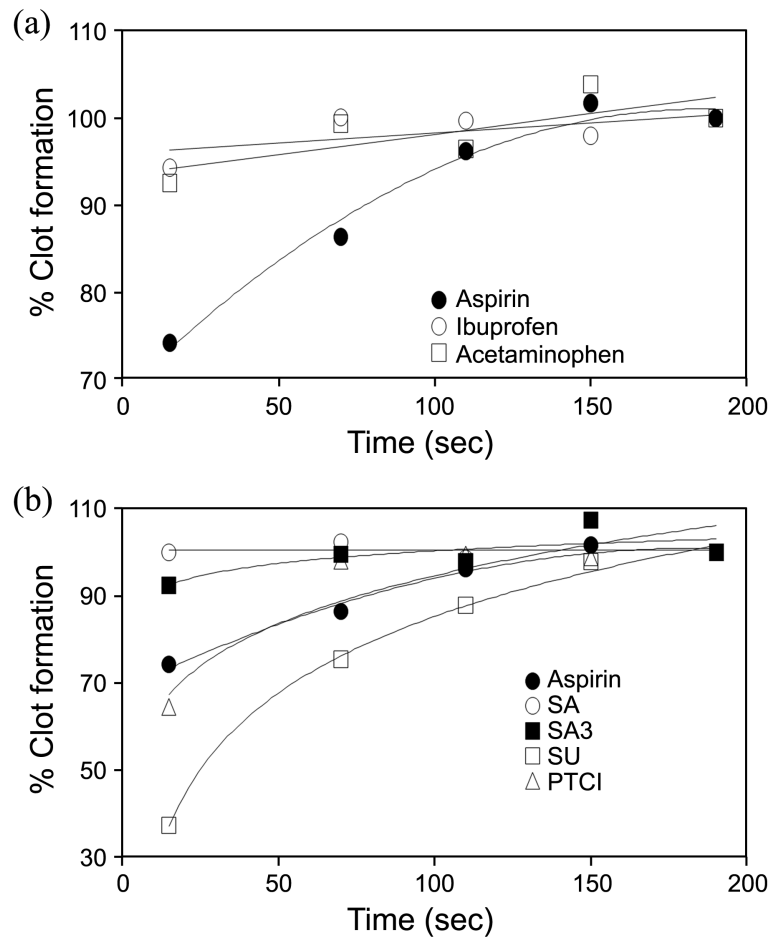

Figure 6. Effects of aspirin, salicylates, and nonsteroidal antiinflammatory drugs on in vitro clot formation. A. Aspirin (-), ibuprofen (O), or acetaminophen $(\square)$ was added to the clot formation assay. B. Same as 6A except aspirin $(\bullet)$, salicyluric acid ( $\square)$, 3-salicylic acid ( $\mathbf{\square})$, or salicylic acid (O). Potato tuber carboxypeptidase inhibitor $(\Delta)$ was used as a positive control.

fibrinolysis model (Table 2, Fig. 5). GA, the salicylate that competitively inhibits TAFIa (as discussed in the previous sections), was the most potent effector of clot lysis. Significantly reduced clot lysis time was also found with $\mathrm{SU}$, which was shown to be an effective blocker of TH/TM-induced TAFI activation and a noncompetitive inhibitor of TAFIa activity. Aspirin and SA were the least active salicylates in the clot lysis model. Potato tuber carboxypeptidase inhibitor (PTCI), a specific inhibitor of TAFI, was used as a positive control in these studies. The reduction of clot lysis time by 


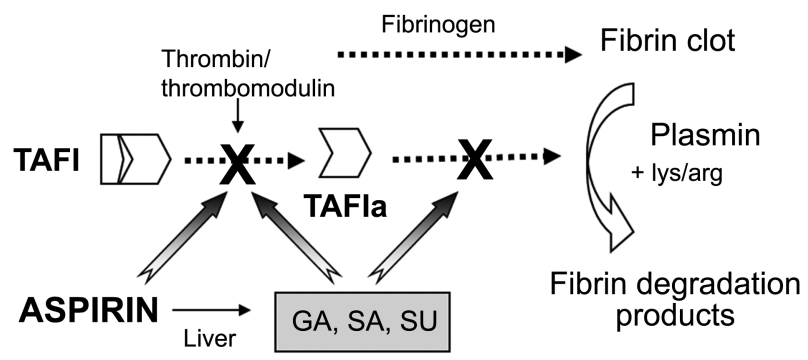

Figure 7. Schematic diagram of the mechanisms by which aspirin, SA and SU enhance fibrinolysis through inhibition of TAFI. TAFIa inhibits plasmin-mediated fibrinolysis by removing lysine groups from partially degraded fibrin (fibrinolysis). Aspirin enhances fibrinolysis by directly inhibiting TAFIa carboxypeptidase activity. SA and SU, the major metabolites of ASA, enhance fibrinolysis by inhibiting thrombin/thrombomodulin (TH/ TM) activation of TAFI and via direct inhibition of TAFIa carboxypeptidase activity.

various salicylate analogs indicates that alterations in fibrinolysis during aspirin therapy may be mediated by TAFI inhibition.

Effect of Salicylates on Clot Formation. TAFI is not only involved in fibrinolytic dissolution of clots but also functions during thrombus formation. We determined whether salicylates also affect thrombus formation in vitro via TAFI inhibition. Thrombin-mediated clot formation was inhibited by aspirin compared to ibuprofen or acetaminophen (Fig. 6(a)). SU showed greater activity than the other noncompetitive salicylate inhibitors, namely, SA3 and SA (Fig. 6(b)). PTCI was used as a positive control in this study.

In the above-mentioned studies, thrombin was used to initiate clot formation. Control studies were done to show that the inhibition of clot formation was due to the effects of the salicylates on TAFI and not on thrombin. Each compound was tested for its ability to inhibit thrombin protease activity. None of the tested compounds inhibited the ability of thrombin to cleave a chromogenic peptide substrate (data not shown). Therefore, the inhibition of clot formation was due to TAFI inhibition and not thrombin inhibition.

\section{Discussion}

The present study showed that aspirin therapy could shift the hemostatic balance toward a profibrinolytic state by inhibiting TAFI, the major protein in plasma that regulates fibrinolysis. Aspirin and a number of its major metabolites inhibited the carboxypeptidase activity of TAFIa (Fig. 2(b) and Table 1). Aspirin, SA, and SU can be classified as noncompetitive inhibitors of TAFIa owing to their ability to inhibit carboxypeptidase activity after a short preincubation period with TAFIa. The rank order of potency was SU $>$ SA $>$ aspirin, indicating that some aspirin metabolites were more potent inhibitors than aspirin itself. GA, unlike the other salicylates, was found to be a relatively potent competitive inhibitor and directly blocked the interaction of TAFIa with the low-molecular-weight substrate used in the fluorescence assay. We conclude that the position of the hydroxyl group(s) on the benzoic acid ring of salicylates could have a profound effect on the drug's interaction with TAFIa.

It is possible that different salicylates bind to distinct site(s) on TAFIa or bind in different orientations at the same site. The mechanism of TAFIa inhibition is likely determined by the mode in which the salicylate binds to TAFIa. TAFIa is a naturally unstable enzyme that rapidly inactivates at $37^{\circ} \mathrm{C}\left(t^{1} / 2=10\right.$ minutes $)$ by undergoing a conformational change to TAFIai. ${ }^{23}$ However, the stability of TAFIa is significantly greater at lower temperatures. In the abovementioned studies, TAFIa was found to rapidly lose enzymatic activity, when preincubated with aspirin and certain various other salicylates at room temperature or at $4{ }^{\circ} \mathrm{C}$. The binding of salicylates to TAFIa may induce similar conformational changes that occur at elevated temperatures, resulting in the rapid loss of activity. Aspirin is more reactive than other salicylates and is known to irreversibly acylate proteins (e.g., COX-1 and fibrinogen) ${ }^{34,35}$ It is possible that the loss of carboxypeptidase activity induced by aspirin may also be due to irreversible TAFIa acylation. The molecular mechanisms of TAFIa inhibition by various salicylates require further study. It is an interesting finding that other NSAIDs, such as ibuprofen and acetaminophen, had little inhibitory effect on TAFIa activity. This finding indicates that TAFIa inhibition is specific for the salicylate class of NSAIDs.

Concentrations of aspirin and its major metabolites can reach very high levels in blood, especially when high doses are taken to treat anti-inflammation and analgesia. The target plasma salicylate concentrations for antithrombotic aspirin therapy are in the range of $100-150 \mu \mathrm{g} / \mathrm{mL} .{ }^{36}$ During highdosage aspirin therapy (0.75-1.5 g/day) for the treatment of inflammation or analgesia, plasma salicylate levels can exceed $400 \mu \mathrm{g} / \mathrm{mL}(>3 \mathrm{mM}) .{ }^{37}$ Salicylates also remain in the blood for long periods of time (approximately a 6-hour half-life). ${ }^{38,39}$ Aspirin had an IC $_{50}$ for TAFIa inhibtion at approximately $600 \mu \mathrm{g} / \mathrm{mL}$. The aspirin metabolic derivatives GA, SA, and SU were more potent than aspirin, with $\mathrm{IC}_{50}$ concentrations of $100-200 \mu \mathrm{g} / \mathrm{mL}$. Upon administration of moderate to high doses of aspirin, salicylates are likely to be present for a sufficient time at the concentrations required to inhibit TAFIa and/or block TAFI activation (see the discussion that follows). Therefore, alterations in fibrinolytic activity associated with aspirin therapy are likely attributable to TAFI inhibition by salicylates under normal physiological conditions.

Some salicylates can also affect TAFI function by inhibiting the TH/TM-induced activation by proteolytic cleavage. The results of the SDS-PAGE analysis (Fig. 4(a)) indicated that SU, the most potent of the TAFIa noncompetitive inhibitors, specifically binds to TAFI site(s) blocking the proteolytic cleavage by TH/TM. When SA and aspirin were added to the TAFI activation reaction, a new $55-\mathrm{kD}$ proteolytic cleavage product was observed. The binding of SA and aspirin to TAFI apparently exposes a new TH/TM proteolytic cleavage site on TAFI. Thus, SA and aspirin 
appear to bind to TAFI in a manner that differs from that of SU. It was difficult to obtain immune-blot of the gel at present, which could be due to the sensitivity of the TAFI antibody, disappearance of the epitope, or low concentrations of new band. The identification of the new cleavage sites after the binding of salicylates to TAFI remains to be determined in future. The new TAFI-related proteolytic cleavage products observed with salicylates might themselves have interesting biological properties that remain to be investigated. Ibuprofen and acetaminophen (Fig. 4(a), lanes 5 and 6 , respectively) had little or no effect on TAFI activation by the TH/TM complex. Thus, salicylates can modulate TAFI-mediated effects on fibrinolysis through 2 mechanisms: (1) retarding activation of TAFI to TAFIa and (2) inhibiting TAFIa carboxypeptidase activity (Fig. 7). Undas et $a l .{ }^{12}$ recently showed that thrombin generation at the wound site is also inhibited by aspirin. Since thrombin is a major proteolytic activator of TAFI, aspirin-induced downmodulation of thrombin generation may also contribute to reduced TAFIa generation.

Carboxypeptidase N (CPN), an enzyme that also removes C-terminal lysine and arginine from proteins, is constitutively expressed in plasma. None of the salicylates that showed activity against TAFI inhibited CPN. In addition, a different pattern of inhibition by salicylates was noted against pancreatic carboxypeptidase B versus TAFI. Thus, TAFI inhibition by various salicylates appears to be specific to TAFI carboxypeptidase activity (data not shown).

It is interesting that SU was shown to exert analgesic and anti-inflammatory effects but was a poor inhibitor of COX$1{ }^{33,34}$ Studies of SU administration in rabbits caused significantly reduced gastric toxicity compared with SA. ${ }^{35}$ Thus, SU and possibly its related derivatives may represent an interesting class of antithrombotic drugs based on its ability to enhance fibrinolysis via TAFI inhibition while not inhibiting platelets or causing gastric mucosal damage owing to its inability to inhibit COX-1.

The salicylates also showed activity in the in vitro fibrinolysis and thrombosis models (Figs. 5 and 6(b)). Aspirin, SU, and GA significantly shortened clot lysis time, a finding that is consistent with the TAFI inhibition in this model. Similarly, the salicylates prolonged clot formation, indicating that TAFI was inhibited by the drug. The experimental results in these biological models lend further support to biochemical studies in which aspirin and aspirin derivatives inhibit TAFI.

In conclusion, we have identified a novel mechanism by which aspirin therapy enhances fibrinolytic activity in the blood via TAFI inhibition. The beneficial effects of aspirin as a prophylactic treatment of cardiovascular disease and its use during antithrombotic therapy can be attributed to a synergistic interaction between enhanced fibrinolysis due to TAFI inhibition and antithrombotic activity by inhibiting COX-1. In addition, the dangerous gastric and cerebral hemorrhagic complications of aspirin therapy may also be attributed to hemostasis imbalances caused by profibrinolytic activity mediated by TAFI inhibition. It would be important to determine whether TAFI or TAFIa admini- stration can counteract the gastric and cerebral bleeding complications associated with high doses of aspirin. These results also suggest that anti-inflammatory and antithrombotic drugs should be screened for anti-TAFI activity to enable full assessment of the full therapeutic consequences of a drug treatment.

Acknowledgments. Authors thank Dr. Richard Hart, American Diagnostica Inc. for discussions and contributions. This work was supported by the GRRC program of Gyeonggi province [GRRCKyungwon 2010-A01, Development of Microfluidic Chip for diagnosis of disease].

\section{References}

1. Antithrombotic Trialists' Collaboration, British Medical Journal 2002, 324, 71-86.

2. Second International Study of Infarct Survival Collaborative Group. Lancet 1988, 2, 349-360.

3. Steering Committee of the Physicians' Health Study Research group. New Eng. J. Med. 1989, 321, 129-135.

4. Mangano, D. T. New Eng. J. Med. 2002, 347, 1309-1317.

5. Roth, G. J.; Majerus, P. W. Proc. Natl. Acad. Sci. 1975, 72, 30733076.

6. Burch, J. W.; Stanford, N.; Majerus, P. W. J. Clin. Invest. 1978, 61, 34-39.

7. Vane, J. R. Nat. New Biol. 1971, 231, 232-235.

8. Baezinger, N.; Bercherer, P. R.; Majerus, P. W. Cell 1979, 16, $967-$ 974.

9. Basinski, A.; Naylor, C. D. J. Am Coll. Cardiol. 1992, 20, 1679.

10. Garabedian, H. D.; Gold, H. K.; Leinbach, R. C.; Svizzero, T. A.; Finkelstein, D. M.; Guerrero, J. L.; Collen, D. J. Am Coll. Cardiol. 1991, 7, 1213-1222.

11. Bjornsson, T. D.; Schneider, D. E.; Berger, H., Jr. J. Pharmacol. Exp. Ther. 1989, 250, 154-161.

12. Undas, A.; Brummel, K.; Musial, J.; Mann, K. G.; Szczeklik, A. Blood 2001, 98, 2423-2431.

13. Green, D.; Davies, R. O.; Holmes, G. I.; Johnson, C.; Kohl, H.; Reynolds, N.; Ts'ao, C. Haemostasis 1983, 13, 394-398.

14. de Gaetano, G.; Carriero, M. R.; Cerletti, C.; Mussoni, L. Biochem. Pharmacol. 1986, 35, 3147-3150.

15. Cattaneo, M.; Chahil, A.; Somers, D.; Kinlough-Rathbone, R. L.; Packham, M. A.; Mustard, J. F. Blood 1983, 61, 353-361.

16. Iacoviello, L.; De Curtis, A.; Amore, C.; D'Adamo, M. C.; Buczko, W.; de Gaetano, G.; Donati, M. B. Eur. J. Pharmacol. 1992, 229, 39-44.

17. Bounameaux, H.; Gresele, P.; Hanss, M.; De Cock, F.; Vermylen, J.; Collen, D. Thromb. Res. 1985, 40, 161-170.

18. Williams, S.; Fatah, K.; Hjemdahl, P.; Blomback, M. European Heart Journal 1998, 19, 1666-1672.

19. He, S.; Bark, N.; Wang, H.; Svensson, J.; Blombäck, M. J. Cardiovasc. Pharmacol. 2009, 53, 24-29.

20. Heylen, E.; Willemse, J.; Hendriks, D. Front. Biosci. 2011, 17, 2427-2450.

21. Marx, P. F.; Verkleij, C. J.; Valls Seron, M.; Meijers, J. C. Mini. Rev. Med. Chem. 2009, 9, 1165-1173.

22. Redlitz, A.; Tan, A. K.; Eaton, D. L.; Plow, E. F. J. Clin. Invest. 1995, 96, 2534.

23. Bajzar, L.; Manuel, R.; Neisheim, M. E. J. Biol. Chem. 1995, 270, 1477.

24. Lapi, F.; Gallo, E.; Giocaliere, E.; Vietri, M.; Baronti, R.; Pieraccini, G.; Tafi, A.; Menniti-Ippolito, F.; Mugelli, A.; Firenzuoli, F.; Vannacci, A. Br. J. Clin. Pharmacol. 2010, 69, 558-560.

25. Meijers, J. C.; Oudijk, E. J.; Mosnier, L. O.; Bos, R.; Bouma, B. N.; Nieuwenhuis, H. K.; Fijnheer, R. Br. J. Haematol. 2000, 108, 
518-523.

26. Watanabe, R.; Wada, H.; Watanabe, Y.; Sakakura, M.; Nakasaki, T.; Mori, Y.; Nishikawa, M.; Gabazza, E. C.; Nobori, T.; Shiku, H. Thromb. Res. 2001, 104, 1-6.

27. Morange, P. E.; Tregouet, D. A.; Frere, C.; Luc, G.; Arveiler, D.; Ferrieres, J.; Amouyel, P.; Evans, A.; Ducimetiere, P.; Cambien, F.; Tiret, L.; Juhan-Vague, I. J. Thromb. Haemost. 2005, 3, 15031510.

28. Meltzer, M. E.; Lisman, T.; de Groot, P. G.; Meijers, J. C.; le Cessie, S.; Doggen, C. J.; Rosendaal, F. R. Blood 2010, 116, 113121.

29. Antovic, J.; Schulman, S.; Eelde, A.; Blombäck, M. Haemophilia 2001, 7, 557-560.

30. Antovic, J. P.; Schulman, S.; An, S. S.; Greenfield, R. S.; Blombäck, M. Scand J. Clin. Lab. Invest. 2004, 64, 745-751.

31. Foley, J. H.; Nesheim, M. E.; Rivard, G. E.; Brummel-Ziedins, K. E. Haemophilia 2011 doi: 10.1111/j.1365-2516.2011.02648.x.
[Epub ahead of print]

32. Mikovic, D.; Woodhams, B. J.; Holmström, M.; Elezovic, I.; Antovic, A.; Mobarrez, F.; Elfvinge, P.; Antovic, J. P. Int. J. Lab. Hematol. 2012, 34, 35-40.

33. Nesheim, M. Chest. 2003, 3 Suppl, 33S-9S.

34. Tosco, P.; Lazzarato, L. Chem. Med. Chem. 2009, 4, 939-945.

35. Bjornsson, T. D.; Schneider, D. E.; Berger, H., Jr. J. Pharmacol. Exp. Ther. 1989, 250, 154-161.

36. Graham, G. G.; Champion, G. D.; Day, R. O.; Paull, P. D. Clin. Pharmacol. Ther. 1977, 22, 410-420.

37. Insel, P. A. Goodman and Gilman's The Pharmacological Basis of Therapeutics, 9th ed.; McGraw-Hill: New York, 1996; pp 617657.

38. Raindford, K. D. Aspirin and Related Drugs; CRC Press: Boca Raton, 2004; 97-137, pp 587-607.

39. Barkin, R. L. Am. J. Ther. 2001, 8, 433-442. 